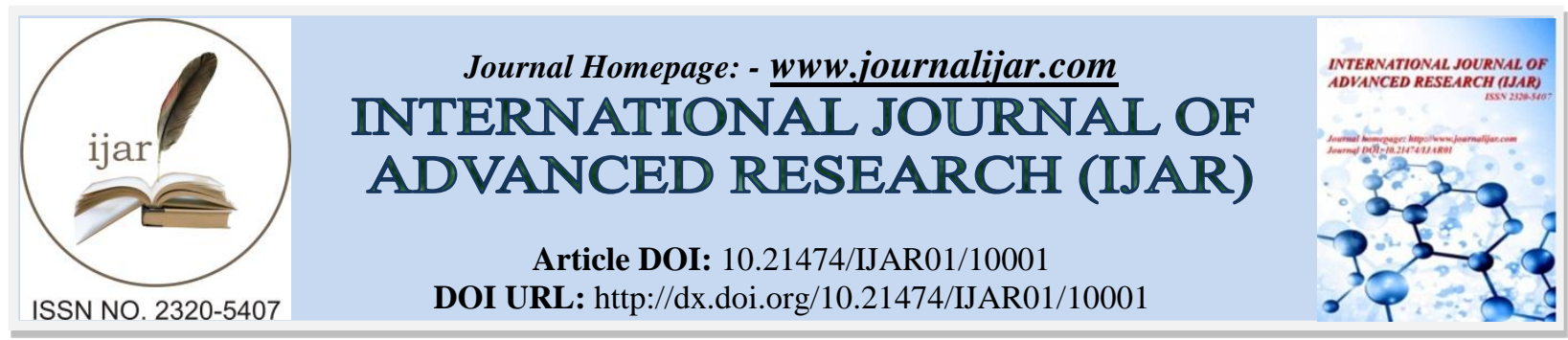

RESEARCH ARTICLE

\title{
TAILORING THE SURFACE TEXTURE OF ZnO NANO STRUCTURES: EVALUATION OF ANTIBACTERIAL ACTIVITY.
}

\author{
S.Tharani, G.Niveditha, R.Mareeswari and T.Nithya. \\ Assistant Professor in Physics, Department of Chemistry and PhysicsNadar Saraswathi College of Arts and Science, \\ Theni-625531, Tamil Nadu.
}

\section{Manuscript Info}

Manuscript History

Received: 05 September 2019

Final Accepted: 07 October 2019

Published: November 2019

\section{Key words:-}

Zinc Oxide NPs, solution Combustion method, XRD,UV, FT-IR, Antibacterial activity.

\section{Abstract}

Zinc Oxide nanoparticles ( $\mathrm{ZnO}$ NPs) were synthesized by solution combustion method where here Zinc Nitrate is used in Powder format and it act as a good oxidizer and malic acid is used as fuel. In UV, the band gap was found to be around $3 \mathrm{eV}$. The X-Ray Diffraction (XRD) exhibit hexagonal structure and confirmed the presences of ZnONPs (JCPDS: 36-1451). The average crystallite size is around $48 \mathrm{~nm}$. FTIR spectra showed the band in the range of $400-600 \mathrm{~cm}^{-1}$, corresponds to stretching vibration mode, which confirms the presence of ZnONPs. The prepared ZnONPs are very effective for gram negative bacteria. The Gram negative bacteria comprises in their membrane structure most of which is thickness of the peptidoglycan layer.

Copy Right, IJAR, 2019,. All rights reserved.

\section{Introduction:-}

Nanotechnology is an emerging field of research and technology dealing with the fabrication and engineering of materials, structures and systems in the nano meter. The concept ideas derived from chemistry, physics, biology merged together to design a novel material with desired properties[1]. Metal Oxides with nano structure have attracted considerable interest in many areas of the technology. ZnO nanoparticles may have many applications such as in the manufacture of electro chromic, films, magnetic materials, p-type transparent conducting films, gas sensors, catalyst, alkaline batteries cathode, and solid oxide fuel cells anode [2]. Several researchers have prepared by various method like sol-gel [3], thermal decomposition [4], solid state reaction [5], precipitation, sono-chemical [6].

Among these methods, solution combustion synthesis is a quick and easy process, with as main advantages the saving of time and energy. This process is used directly in the production of high purity, homogeneous ceramic oxide powders. Solution Combustion Synthesis (SCS) is a versatile process for the synthesis of a wide size range of particles including nanometer size powder as reported by Patile et al [7]. It uses a metal nitrate as oxidizing agent and an organic fuel as reducing agent in oxidation/reduction reactions that occur as the catalyst is synthesized. Although the heat obtained can help to decompose metal nitrate and salts, a primary heat is required to initiate the reactions so that they can continue the process without additional energy by Kopp Alves et al [8].

Depending on the type of precursors, as well as on the conditions used for the process organization, the SCS may occur as either volume or layer-by-layer propagating combustion modes. From a chemistry standpoint this result is not surprising because it is well-known that decomposition of metal nitrates leads to the formation of corresponding oxides. Large volumes of gases are generated during this auto-ignition process, making find powder as the resultant 
product. It is very important to develop methods for the synthesis of nickel oxide nanoparticles in which the particle size and the crystal structure of the products can be controlled [9].The materials like nickel, copper, silver, zinc present high antibacterial activity, low toxicity, chemical stability, Thermal resistance compared to organic antibacterial agents [10]. In the present study, Malic acid was used as fuel in Solution Combustion Method to synthesize ZnO NPs. It's optical, structural and antibacterial activities were investigated.

\section{Synthesis Of Zno Nps}

The preparation of ZnO NPs, Zinc Nitrate and Malic acid was taken as starting materials. The Stoichiometric compositions of the solution components (fuels and oxidizer) were calculated according to the principle of propellant chemistry, keeping the oxidizer to fuel ratio as unity. Stoichiometric amount of Zinc Nitrate was dissolved in de-ionized water and then starch was added in to it. The solution was kept in the furnace at $300^{\circ} \mathrm{C}$.

$$
\mathrm{Zn}\left(\mathrm{NO}_{3}\right)_{2(\mathrm{c})}+0.83 \mathrm{C}_{4} \mathrm{H}_{6} \mathrm{O}_{5(\mathrm{c})}+\mathrm{O}_{2(\mathrm{~g})} \quad \mathrm{ZnO}_{(\mathrm{c})}+\mathrm{N}_{2(\mathrm{~g})}+3.32 \psi \mathrm{CO}_{2(\mathrm{~g})}+2.4 \psi \mathrm{H}_{2} \mathrm{O}_{(\mathrm{g})}
$$

The situation of an equivalent stoichiometric ratio which implies that the oxygen content of nickel nitrate can be completely reacted to oxidize/consume Malic acid exactly. As a result, $\mathrm{ZnO}$ product and gases of $\mathrm{CO}_{2}, \mathrm{H}_{2} \mathrm{O}$ and $\mathrm{N}_{2}$ can be formed directly from the reaction between fuel and oxidizer without the necessary of getting oxygen from outside.

\section{Characterization}

The X-ray diffraction (XRD) patterns were recorded on PAN analytical X-ray diffractometer using $\mathrm{Cu}$ Ka radiation $(\lambda=0.1542 \mathrm{~nm})$ operated at $50 \mathrm{KV}$ and $100 \mathrm{~mA}$. The experiments performed in the diffraction angle range of $2 \theta=20$ $80^{\circ}$.Shimadzu $1700 \mathrm{UV}-$ Visible spectrophotometer was used to carry out the optical measurements. The Shimadzu IR affinity-1 Fourier Transform Infrared spectrometer was used to carry out vibrational studies and Energy Dispersion Spectroscopy (EDS) (200KeV JEOLJEM2100).

\section{Assay For Antimicrobial Activity Of Zno Nps Against Microorganism}

The antimicrobial activity of $\mathrm{ZnO}$ NPs were evaluated against gram negative, Klebsiella pneumonia, Acinetobacter baumannii, Escherichia coli, Vibrio cholerae, Shigella flexneri by their agar disc diffusion method. Mueller Hinton agar plates were spread with $100 \mu \mathrm{L}$ of actively grown broth cultures of the respective test bacteria and were allowed to dry for 10 minutes. The plates were incubated for 48 hours at $37^{\circ} \mathrm{C}$. The development of the inhibition zone around the extract loaded discs was recorded.

\section{Results And Discussion:- \\ X-ray diffraction spectroscopy (XRD)}

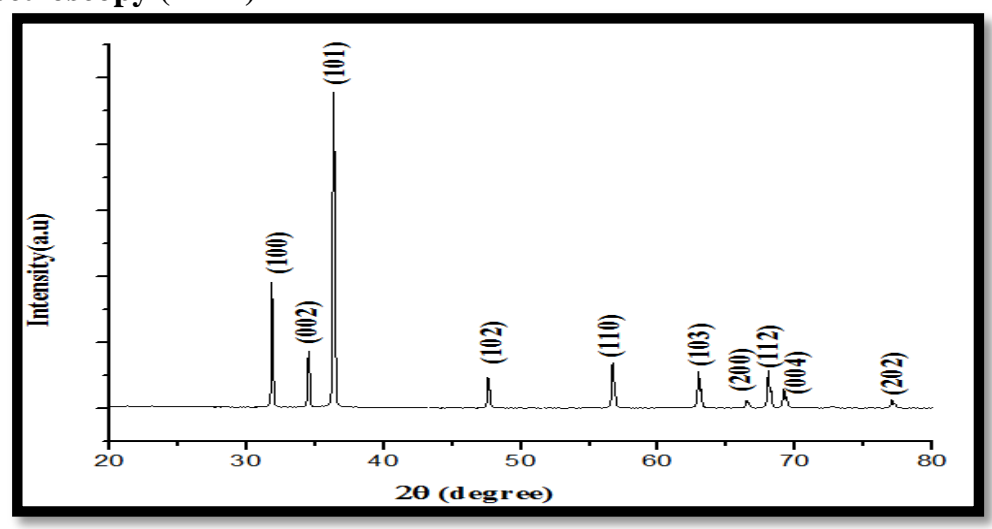

Fig1:-XRD spectra for ZnO NPs

Fig.1 shows the XRD spectra of $\mathrm{ZnO}$ Nanoparticles. The five diffraction peaks of the XRD pattern of ZnO NPs were observe data $31.88^{\circ}, 34.53^{\circ}, 36.36^{\circ}, 47.65^{\circ}, 56.68^{\circ}, 62.92^{\circ}, 66.43^{\circ}, 68.01^{\circ}, 69.15^{\circ}, 77.03^{\circ}$ which correspond to (100), (002), (101) , (102), (110), (103), (200),(112),(004) and (202)diffraction planes respectively (ICDD: 36-1451).All diffraction peaks of $\mathrm{ZnO}$ correspond to the cubic structure .The volume of the cell were $47.63\left(\mathrm{~A}^{0}\right)^{3}$ The average particle size of the ZnO NPs were calculated using the Debye Scherrer equation, 


\section{$\mathbf{D}=\mathbf{K} \lambda / \boldsymbol{\beta} \cos \theta$}

where, $D$ is the crystallite size $(\mathrm{nm}), \lambda$ represents wavelength of X-ray source $\left(0.15406 \mathrm{~A}^{\circ}\right), \beta$ is the full width at half maximum of the diffraction peak (FWHM) in radians, $\mathrm{K}$ is the Scherrer constant with value from 0.9 to $1, \theta$ is the Bragg angle in (degree) . The lattice constant value are also calculated and very close to the standard data is a= $3.249 \mathrm{~A}^{0}, \mathrm{c}=5.206 \mathrm{~A}^{0}$. The average particle size found to be about $48 \mathrm{~nm}[11]$.

\section{Williamson-Hall method}

Numerous experiments have shown that strain broadening caused by dislocations can be well described by a special logarithmic series expansion of the Fourier coefficients of Bragg reflection peak profiles. It can be calculated from the formula, $\varepsilon_{\mathrm{str}}=\beta / \tan \theta$.

W-H equation may be expressed in the form,

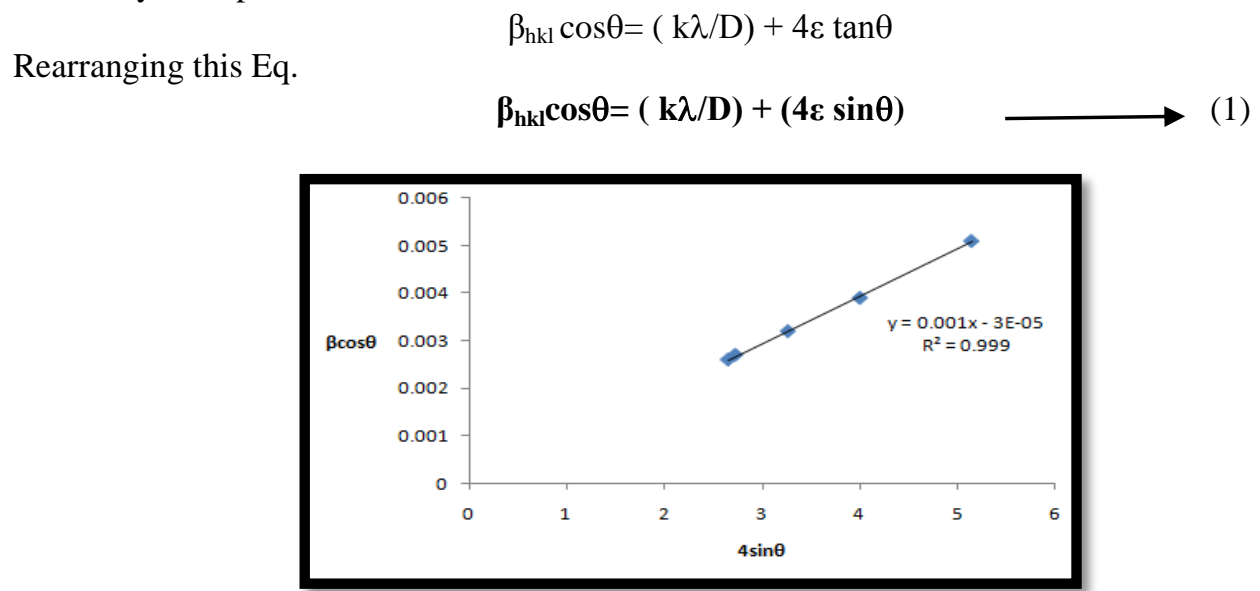

Fig 2:-W-H plot analysis of $\mathrm{ZnO}$ nanostructure assuming UDM. Fit to the data, the strain is extracted from the slope.

where, $\varepsilon$ is the strain associated with the Nanoparticles A plot is drawn between $\beta \cos \theta$ and $4 \sin \theta$ for $\mathrm{ZnO} N P s$, the slop of the line gives the strain $(\varepsilon)$ and intercept $(k \lambda / \mathrm{D})$ of the line gives grain size (D).

The term $\beta \cos \theta$ was plotted with respect to $4 \sin \theta$ for the prepared orientation peaks of $\mathrm{NiO}$ nanostructure. Fig .2 shows the results of $\mathrm{W}-\mathrm{H}$ analysis for $\mathrm{ZnO}$ is positive strain and is found to have the value 0.001 . The line broadening of cubic $\mathrm{ZnO}$ was due to small crystallite size and lattice strain. However, the strain arising from $\mathrm{W}-\mathrm{H}$ model is very small and has negligible effect on peak broadening.

\section{Fourier Transforms infrared spectroscopy (FT-IR)}

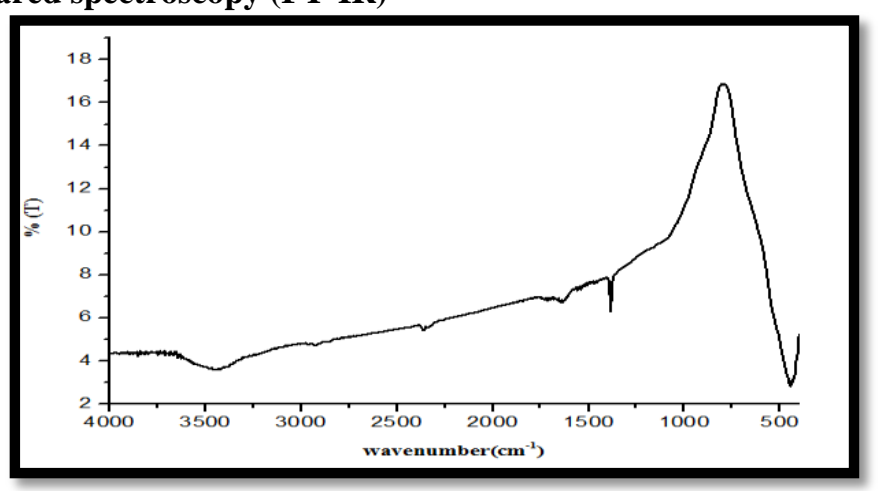

Fig3:-FTIR spectra of ZnO NPs

Fig.3 shows the FTIR spectra of combustion product of $\mathrm{ZnO}$ NPs. The FTIR spectrum was observed over the frequency range $500-4000 \mathrm{~cm}^{-1}$. The broad band in the region of $400-600 \mathrm{~cm}^{-1}$ is assigned to $\mathrm{Zn}-\mathrm{O}$ stretching vibration mode. The prepared sample shows the characteristics band of $3474 \mathrm{~cm}^{-1}$ was attributed to O-H stretching 
of hydrogen bonded water on the surface of NPs and the band at $1635 \mathrm{~cm}^{-1}$ is assigned to $\mathrm{H}-\mathrm{O}-\mathrm{H}$ bending vibrations mode presented due to the adsorption of water in air during the preparation of FTIR sample disks in an open air atmosphere. The band at $1382 \mathrm{~cm}^{-1}$ is attributed to $\mathrm{O}-\mathrm{C}=\mathrm{O}$ symmetric and asymmetric stretching vibrations [12].

\section{UV-Visible Spectroscopy (UV-Vis)}

The absorption spectra of $\mathrm{ZnO}$ nanoparticles observed in the UV region are depicted in Fig.5(a).The absorption peak shoulder on set peaks are located at $247 \mathrm{~nm}$ respectively. The

$$
\alpha h v=A\left(h v-E_{g}\right)^{n / 2}
$$

Where, $\mathrm{h} v$ is the photon energy is the absorption coefficient, A is a constant and $\mathrm{n}=1$ for direct transition. Fig. 4 (b) shows the plot of $(\alpha h v)^{2}$ versus $\mathrm{h} v$ for the $\mathrm{ZnO}$ sample. From the plot, the band gap value of the $\mathrm{ZnO} \mathrm{NPs}$ was calculated to be $3 \mathrm{eV}$. This is lower when compared to bulk $\mathrm{ZnO}(3.37 \mathrm{eV})$. The lower band bap value of $\mathrm{ZnO}$ nano particles exhibits the nature of blue shift due to quantum confinement effect in the nano scale region [13].
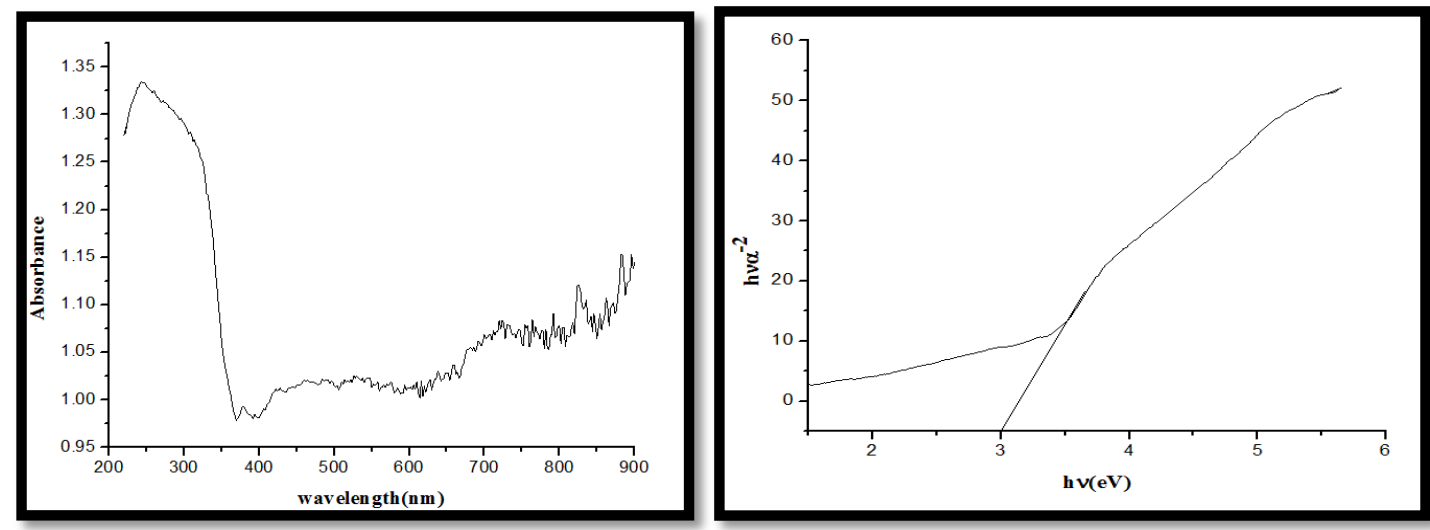

Fig 4:-(a) optical absorption spectra of ZnO NPs and (b) plot of $(\alpha h v)^{\wedge 2}$ versus $h v$.

\section{Energy Dispersive Spectroscopy (EDS)}

The energy dispersive spectra of the prepared ZnO NPs were shown in Fig.5. It confirms the presence of Zinc Oxide. The signal of $\mathrm{Cu}$ originated from the carbon coated $\mathrm{Cu}$ grid.

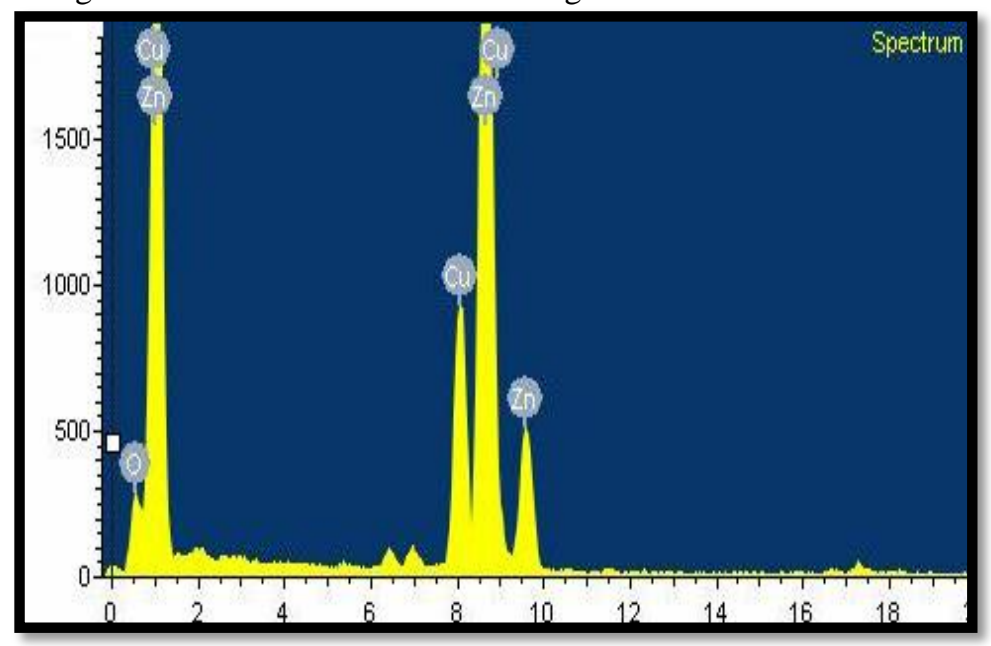

\section{Antibacterial activity}

Fig 5:-Energy dispersion spectra of $S_{1}, S_{2}$ and $S_{3}$

In this study, the antimicrobial activity against various microorganisms (gram negative), Klebsiella pneumonia, Acinetobacter baumannii, Escherichia coli,Vibrio cholera,Shigella flexneri were evaluated. 


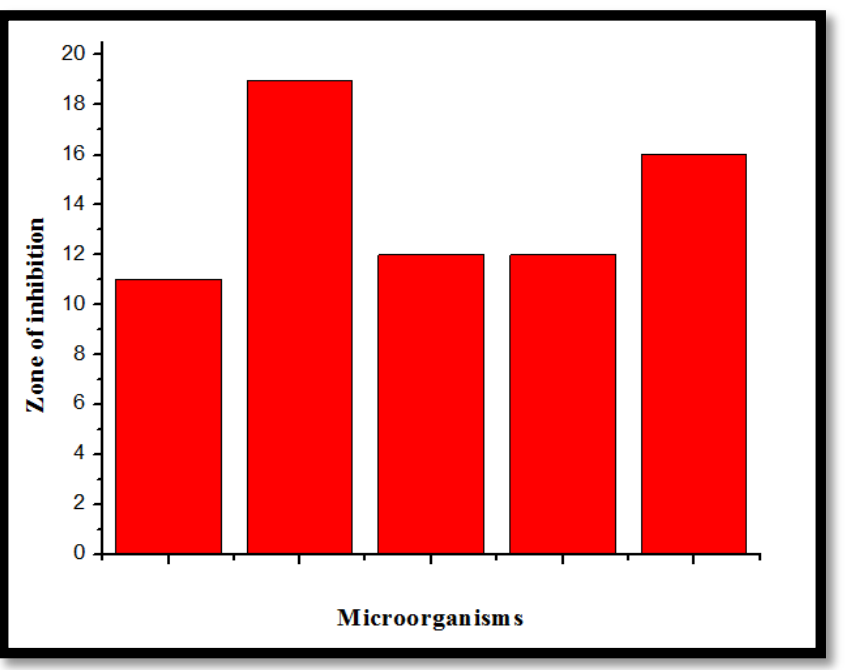

Fig 6:-The plot represent antibacterial effect of $\mathrm{ZnO}$ nanostructure

\begin{tabular}{|l|c|}
\hline \multicolumn{1}{|c|}{ Microorganisms } & Zone of inhibition(mm) \\
\hline Klebsiellapneumoniae & 11 \\
\hline Acinetobacter baumannii & 19 \\
\hline Escherichia coli & 12 \\
\hline Vibrio cholera & -12 \\
\hline Shigella flexneri & 16 \\
\hline
\end{tabular}

Table 1:-Antibacterial efficacy result of $\mathrm{ZnO} \mathrm{NPs}$

Table 1 show that Antibacterial activity is studied for ZnONPs using disc diffusion method. The prepared ZnO NPs are very effective for gram negative bacteria.The Gram negative bacteria comprises in their membrane structure most of which is thickness of the peptidoglycan layer.The antibacterial activity of ZnO NPs was quantitatively assessed on the basis of zone of inhibition and compared with standard antibiotic[14].

\section{Conclusion:-}

The ZnO NPs were synthesized by solution combustion method using Malic acid as fuel. The EDS measurement shows the formation of $\mathrm{ZnO}$ NPs. The XRD shows that the prepared $\mathrm{ZnO}$ NPs having hexagonal structure. It was observed that Zinc oxide nanoparticles can be used in medical field due to their efficient antimicrobial function.

\section{REFERENCES}

1. ShreyashHadke,MadhuTeluguKalimila,ShashwatRathkanthiwar,ShivaniGour,CeramicInt.Sci.Direct 42(2015) 14949-14957.

2. A.Manikandan, J.JudithVijaya, L.JohnKennedy, Physica E 49(2013) 117-123.

3. M.Kumar Rath, S.Kumar Acharya, B.H. Kim et al, Matter.Lett ( 2011),65,955-958.

4. X.J.Zhang,D.G.Zhang,X.M.Ni,J.M.Song,H.G.Zheng,Nanopart.Res.10(2008) 839-844.

5. C.C.Vidyasagar, Y.ArthobaNaik, T.G.Venkatesha, Nano Micro Lett.4(2012) 73-77.

6. R.V.Kumar, Y.Diamant, A.Gadanken,Chem.Mater.12(2000) 2301-2305.

7. A.Kopp Alves et al Springer-Verlag Berlin Heig.berg (2013)

8. Alves,C.,Oliveira,A.Carneiro,S.Silva,A.Andrade.S.V.Torres,FuelprocessTech(2013)106,102

9. 107.

10. M.Salavati-Niasari,F.Mohandes,F.Davar,M.Mazaheri,M.MonemzadeshandN.Yavarinia, Inorg.chimia.Acta. (2009) 3691-3697

11. Singanahally T.Aruna,Aloxander S.Mukasyan,Current Opinion in solid stat and Mater Sci12(2008) 44-50.

12. Yuvakkumar R,Suresh J,Saravana Kumar B.Nathanaeld AJ,Honga SI,Rajendran Molecular and Biomole Spectro 137 (2015) 250-258.

13. M.Hasanpoor,M.Aliofkhazraei,H.Delavari,Pro.Mater.Sci. 11(2015) 320-325.

14. RatiramGomajichaudhry,JayA.Tanna,NileshV.Gandhare,AlokR.Rai,AdvanceMater.letter (2015) 611,990-998. 